\title{
Data Sharing Across Jurisdictions Using ESSENCE Federated Queries
}

\author{
Wayne Loschen*, Rekha Holtry and Sheri Lewis
}

Johns Hopkins University Applied Physics Laboratory, Laurel, MD, USA

\section{Objective}

The objective of this presentation is to describe the new federated query capability in ESSENCE and describe how this could affect public health practice in the future. Specifically, this presentation will describe how a federated set of disease surveillance systems across the country could help improve national disease surveillance situational awareness along with its potential to connect non-ESSENCE systems in the future for even more complete coverage. It will also describe how this capability is different than other data sharing projects that attempt to centralize data, but how there is room for both to benefit from each other.

\section{Introduction}

There are currently over 25 installations of ESSENCE across the US. Among these, there are 3 instances of multi-jurisdictional implementations. These include a centralized regional system in the National Capital Region for MD, DC, and VA, a Missouri system that includes hospitals and users from the St. Louis area in Illinois, and soon the National Syndrome Surveillance Program (NSSP) version of ESSENCE which will centralize data from many jurisdictions. While each of these systems provides valid ways to share data across jurisdictions, they require data to be sent to another jurisdiction. There are some jurisdictions which have legal or philosophical or technical issues with these types of data sharing arrangements. Programs like Distribute attempted to solve this by only sharing pre-aggregated data. This caused issues though for surveillance of new and emerging issues that requires a more ad-hoc query capability. This gap can be filled with a locally-ran system that has the ability to perform queries into remote systems and perform a federated query across other jurisdictions.

\section{Methods}

A new capability has been developed for ESSENCE systems that allow local administrators to publish their data sources for use by another jurisdictions ESSENCE users. When this occurs, all data remains in the local ESSENCE system, but other ESSENCE users can send requests to that system. These requests, such as time series or data details requests, can be accepted or denied based on access control permissions on an individual user or jurisdiction basis. Using this new technology that can connect ESSENCE systems to other ESSENCE systems, it will become possible technically to query for new and emerging disease trends by performing ad-hoc queries across many different jurisdictions. Additionally, any current multijurisdictional system, such as the future NSSP ESSENCE system, would also be able to technically participate in this new federate query framework. This provides local systems, which may want a customized ESSENCE system, the ability to share with multijurisdictional systems, which may provide ESSENCE to jurisdictions that can't support a local system.

\section{Results}

While the technology to perform federated queries across ESSENCE systems has now been developed, it will not be deployed into an active system until late 2015. Early results on how the system has performed can be presented then. However, the potential ramifications of having this technology will be discussed along with potential future enhancements to support non-ESSENCE disease surveillance system federated query support.

\section{Conclusions}

Data and information sharing is not simple. Many jurisdictions desire it and many projects have succeeded in many aspects of it. This is another piece of the data and information sharing puzzle that will allow jurisdictions that prefer not to centralize their data to still participate in a data and information sharing collaboration.

Keywords

Data Sharing; Information Sharing; ESSENCE; Federated Queries

\section{*Wayne Loschen}

E-mail: Wayne.Loschen@jhuapl.edu 